\title{
The genetic architecture of fitness in a seed beetle: assessing the potential for indirect genetic benefits of female choice T Bilde ${ }^{* 1,2}$, U Friberg ${ }^{1,3}$, AA Maklakov ${ }^{1,4}$, JD Fry ${ }^{5}$ and G Arnqvist ${ }^{1}$
}

Address: ${ }^{1}$ Animal Ecology/Department of Ecology and Evolution, Evolutionary Biology Centre, University of Uppsala, Uppsala SE-753 32 , Sweden, ${ }^{2}$ Department of Biological Sciences, Ecology and Genetics, University of Aarhus, 8000 Aarhus C, Denmark, ${ }^{3}$ Department of Ecology, Evolution and Marine Biology, University of California, Santa Barbara, California 93106-9610, USA, ${ }^{4}$ School of Biological, Earth and Environmental Sciences, The University of New South Wales, Kensington, Sydney 2052, Australia and ${ }^{5}$ University of Rochester, Department of Biology, Rochester, New York 14627-0211, USA

Email: T Bilde* - trine.bilde@biology.au.dk; U Friberg - friberg@lifesci.ucsb.edu; AA Maklakov - A.Maklakov@unsw.edu.au; JD Fry - jfry@mail.rochester.edu; G Arnqvist - goran.arnqvist@ebc.uu.se

* Corresponding author

Published: 26 October 2008

BMC Evolutionary Biology 2008, 8:295 doi:10.1 I86/I47I-2।48-8-295
Received: 2 May 2008

Accepted: 26 October 2008

This article is available from: http://www.biomedcentral.com//47I-2/48/8/295

(c) 2008 Bilde et al; licensee BioMed Central Ltd.

This is an Open Access article distributed under the terms of the Creative Commons Attribution License (http://creativecommons.org/licenses/by/2.0), which permits unrestricted use, distribution, and reproduction in any medium, provided the original work is properly cited.

\begin{abstract}
Background: Quantifying the amount of standing genetic variation in fitness represents an empirical challenge. Unfortunately, the shortage of detailed studies of the genetic architecture of fitness has hampered progress in several domains of evolutionary biology. One such area is the study of sexual selection. In particular, the evolution of adaptive female choice by indirect genetic benefits relies on the presence of genetic variation for fitness. Female choice by genetic benefits fall broadly into good genes (additive) models and compatibility (non-additive) models where the strength of selection is dictated by the genetic architecture of fitness. To characterize the genetic architecture of fitness, we employed a quantitative genetic design (the diallel cross) in a population of the seed beetle Callosobruchus maculatus, which is known to exhibit post-copulatory female choice. From reciprocal crosses of inbred lines, we assayed egg production, egg-to-adult survival, and lifetime offspring production of the outbred FI daughters (FI productivity).
\end{abstract}

Results: We used the bio model to estimate six components of genetic and environmental variance in fitness. We found sizeable additive and non-additive genetic variance in $F_{1}$ productivity, but lower genetic variance in egg-to-adult survival, which was strongly influenced by maternal and paternal effects.

Conclusion: Our results show that, in order to gain a relevant understanding of the genetic architecture of fitness, measures of offspring fitness should be inclusive and should include quantifications of offspring reproductive success. We note that our estimate of additive genetic variance in $F_{1}$ productivity $\left(C V_{A}=14 \%\right)$ is sufficient to generate indirect selection on female choice. However, our results also show that the major determinant of offspring fitness is the genetic interaction between parental genomes, as indicated by large amounts of non-additive genetic variance (dominance and/or epistasis) for $F_{1}$ productivity. We discuss the processes that may maintain additive and non-additive genetic variance for fitness and how these relate to indirect selection for female choice. 


\section{Background}

Female choice is defined as when a trait in females (behavioral, morphologic, physiological) biases reproductive success among males towards certain male phenotypes over others [1]. The evolution of adaptive female mate choice relies on variation in the quality of potential mates, and the ability of females to select sires of the highest quality [2]. While direct benefits of mate choice provide females with immediate benefits in the form of resources, indirect benefits of mate choice are acquired through enhanced genetic quality of offspring [3-7]. Female choice for high quality males may result from either pre-copulatory mating biases [2] or post-copulatory processes in the female reproductive tract that lead to differential use of male gametes for fertilization [8]. Models for the evolution of female choice based on indirect genetic benefits are usually grouped into good genes models, where alleles with additive effects confer fitness benefits, and compatibility models, where the combining ability of specific alleles (i.e., epistasis) of the male and female genomes determines fitness $[2,9,10]$. These models, thus, rely on different types of genetic variation for fitness.

Female choice may result from indirect selection, by a process known as the "good genes" process [11]. This results from an association, by linkage disequilibrium, between alleles that determine female preference at one locus and those that affect viability at other loci. Theoretical models have shown that such associations develop largely as a result of additive effects of viability loci [12]. Epistasis contributes little if anything to this process, simply because recombination tends to disassociate coadapted alleles across viability loci [12]. Similarly, good genes models that have considered dominance variation, in the form of recessive deleterious mutations, show that dominance have little if any effect on the good genes process $[13,14]$.

Genetic compatibility scenarios are based on females deriving indirect genetic benefits from pairing with genetically compatible males $[6,9,10,15]$. Here, compatibility refers to cases where the fitness effects of an allele depend on either its homologue (dominance and over-dominance) or on a specific allele at another locus (epistasis) and these models rely on non-additive genetic variation across the genome [15-17]. Female choice for genetically compatible mates, thus, do not and can not involve indirect selection (see above), but would result from direct selection among females for indirect genetic benefits. We note that such processes are not expected to lead to the "run-away" selection that has been suggested to result in evolutionary exaggeration or elaboration of male traits. Further, although good genes and compatibility models for the evolution of female choice are distinct in theory, they may not be mutually exclusive in the sense that addi- tive and non-additive genetic effects are not statistically independent [16].

For an association between female preference genes and viability/fitness genes to develop, sufficient levels of additive genetic variation for fitness must be present in the population. The classical interpretation of Fisher's fundamental theorem [18] holds that additive genetic variation for fitness is effectively depleted by directional selection $[16,19]$ : alleles conferring fitness advantages should become fixed in the population while those that reduce fitness should gradually be lost $[20,21]$. The evolvability of fitness should therefore be very low in a stable environment. This general scenario has been supported by numerous studies of heritability, collectively suggesting that the heritability of traits closely related to fitness (e.g. survival and fecundity) tends to be lower than that of morphological and behavioral traits, which generally are less closely associated with fitness [16,22-24]. However, the narrow sense heritability $\left(h^{2}\right)$ of a trait represents the ratio of additive genetic variance $\left(V_{A}\right)$ to the total phenotypic variance $\left(V_{P}\right)$ and the heritability of traits largely affected by non-additive effects or environmental factors will thus by definition be low [25-28]. Heritability estimates may therefore often be inappropriate for quantifying the long-term evolutionary potential of a trait (see discussion in [21]) and they are entirely inadequate for detecting epistatic interactions. Instead, Houle (1992) argued that the coefficient of additive genetic variance $\left(C V_{A}\right)$, where additive genetic variance is scaled by the trait mean, is a more appropriate measure when comparing the evolutionary potential of quantitative traits. When comparing $h^{2}$ and $C V_{A}$ across traits in Drosophila, Houle (1992) found that $C V_{A}$ for fitness-related traits were actually higher than those for morphological traits (see also [29] for a similar finding). One obvious explanation for this is the fact that traits closely related to fitness should be more polygenic and should thus capture genetic variation across many loci [30]. Fitness related traits also show a high mutational input to genetic variance [31], again presumably reflecting their polygenic nature [32]. The same scaling can be applied to other components of genetic variance (e.g. non-additive components [D. Houle, pers. comm.]).

Non-additive genetic effects caused by interactions among alleles within and between loci (i.e., dominance and epistasis) may be important determinants of fitness [28,33-35]. Given that quantitative fitness traits are polygenic, we would expect that fitness related traits would harbor fairly high absolute levels of non-additive genetic variation $[21,26,36,37]$.

The general importance of indirect genetic benefits for the evolution of female choice is still debated [11-13,38]. Further, the relative roles of the good genes process and 
genetic compatibility scenarios remain largely unexplored $[6,11,15,17]$. One way to address these questions is to partition and quantify additive and non-additive genetic variance for fitness, since the nature of the potential indirect genetic benefits of mate choice depends critically on the genetic architecture of fitness. While additive genetic variance of fitness has been studied in a few populations of birds and mammals $[22,26,39,40]$ data on the genetic architecture of fitness or fitness related traits are scarce [41-44].

The purpose of this study was to investigate the genetic architecture of fitness, with the specific goal of estimating additive and non-additive (composite of dominance and epistasis) genetic variance components. This was achieved by employing a quantitative genetic design, the diallel cross $[34,35,45]$, that allows additive and non-additive genetic variance components to be estimated using the bio model [41,45-49]. As a model system, we used the seed beetle Callosobruchus maculatus (Coleoptera, Bruchidae). This species is moderately polyandrous and is known to exhibit post-copulatory female choice [50-54]. The species is also well suited for establishing the homozygous discrete genotypes necessary for conducting the reciprocal cross [34]. We first created inbred lines, with an estimated inbreeding coefficient of 0.89 , from a large and outbred population. In reciprocal crosses of these genotypes, we then assayed egg production, egg-toadult survival and lifetime offspring production of the F1 daughters. Hence, we obtained integrative measures of offspring quality that reflect the net effect of the parental genetic contribution to offspring fitness. Such data are scarce but vital for understanding the potential fitness consequences of mate choice $[7,55]$.

\section{Methods}

Our study animal was a laboratory population of C. maculatus (F.) (Coleoptera: Bruchidae), obtained from C.W. Fox, University of Kentucky, Lexington. This population originated from infested mung beans (Vigna radiata) collected in Tirunelveli, India in 1979 [56], and has been maintained in the laboratory for more than 100 generations at population sizes $>1500$ individuals. Hence, this population is unlikely to have gone through a bottleneck that would have depleted the genetic variation $[56,57]$ (see also [58]). In addition, this C. maculatus population was well suited because females eliminate competition among progeny within host beans by laying only one egg on each bean $[56,57,59]$.

Young C. maculatus larva bores into the bean where it completes development, emerging as an adult after 24-26 days at a temperature of $25^{\circ} \mathrm{C}$. We kept beetles on organically grown Mung beans in incubators at $25^{\circ} \mathrm{C}$ and a relative humidity of approx. $60 \%$. Virgin beetles for our experiments were obtained by isolating individual beans containing single larvae/pupae prior to eclosure. Inbred lines were founded in December 2004, in the following manner. Virgin females $(\mathrm{N}=215)$ were mated with one male each and then placed individually in vials $(30 \mathrm{ml})$ containing more than 100 Mung beans for oviposition. Prior to eclosure, beans containing single larvae were isolated and a single virgin female was mated once to a full sib brother and then confined individually in a container $(30 \mathrm{ml})$ with an excess of Mung beans for oviposition. This procedure was repeated for 10 generations. The estimated inbreeding coefficient after 10 generations of full sib matings is $F=0.89$.

Within each line and each generation, the above procedure was performed in parallel using two females each mated to one full sib brother to reduce the risk of stochastic reproductive failure and hence reduce the rate of line loss. Nonetheless, approximately $60 \%$ of the lines were lost during the inbreeding process. This line loss represents a source of selection that could potentially bias the estimates of genetic variation. The likely effect would be to deflate genetic variance estimates, which would render our variance estimates conservative. To increase the variance estimates, the selection process would have had to cause selective extinction of lines with intermediate breeding values, leaving lines with high and low values, which seems unlikely.

\section{The diallel reciprocal cross}

From a set of 19 lines we employed a full diallel design with reciprocal crosses [34,45]. From each line, we isolated virgin males and females and then paired females from each line with males from each of the 19 lines. Each focal pair was placed in a vial with excess beans as described above. Preliminary observations suggested that virgin females accepted the first mating regardless of male genotype, hence, we assume here that all pairs would mate. In total, we performed 361 line crosses (342 between-line crosses and 19 within-line crosses). Between-line crosses were each replicated three (in some cases four) times $(\mathrm{N}=3(4)$ focal pairs per cross $)$ and within-line crosses were replicated four times $(\mathrm{N}=4$ focal pairs per cross). From each focal pair, we recorded the following four variables: (1) the total number of eggs produced (lifetime egg production); (2) the total number of adult offspring produced (lifetime offspring production); and (3) the egg-to-adult survival of offspring, i.e. juvenile fitness (number of offspring produced divided by the number of eggs laid). Total offspring production was obtained by isolating all beans with eggs until offspring eclosed. We then randomly selected 4-6 daughters from each focal pair and paired each virgin daughter with a randomly selected outbred male following the procedure described above. These males came from crosses between 
inbred lines (overall from 82 inbred lines, see procedure above), and males never originated from overlapping parental lines as the females. With this procedure, we yielded a measure of (4) the lifetime offspring production of F1 daughters of each focal pair (F1 productivity). With this design, we did not expect male genotype to contribute significantly to genetic variance in F1 productivity. This assumption was confirmed by statistical analyses showing that the genotype of the F1 males explained only a very small proportion of the variance $(<2 \%)$ in F1 productivity. Hence, male genotypic effects were therefore subsequently disregarded.

Unmated C. maculatus females produce some unfertilized eggs when a suitable substrate is available, but some females in our experiment nevertheless failed to produce eggs. For all analyses reported below, we ran parallel models using both the full data set and a data set excluding females with no egg/offspring production. Both types of data yielded very similar variance component estimates. However, because the restricted data set better conformed to the assumption of our inferential models, we present these results below (Table 1). Here, pairs that did not produce any eggs were coded as missing values. Egg-to-adult survival was transformed prior to analyses using the arcsine square root transformation.

\section{Variance component estimates}

We estimated variance components using the bio model of Cockerham \& Weir (1977) and Lynch \& Walsh (1998). For the first three traits (excluding $\mathrm{F}_{1}$ productivity), the model equation was

$$
Z_{i j k}=\mu+N_{i}+N_{j}+T_{i j}+M_{j}+P_{i}+K_{i j}+R_{k(i j)} .
$$

Here, $Z_{i j k}=$ the trait value from the $k$ 'th replicate cross between line $i$ males and line $j$ females, and $\mu=$ mean phenotypic value of the population. The other terms are assumed to be mutually independent, normally distrib- uted variables with mean zero, representing the following random effects:

$N_{i}$ and $N_{j}=$ haploid nuclear contributions from parental lines $i$ and $j$ (independent of sex).

$T_{i j}=$ interaction between haploid nuclear contributions.

$M_{j}=$ maternal genetic and environmental effects of line $j$ when used as dams.

$P_{i}=$ paternal genetic and environmental effects of line $i$ when used as sires.

$K_{i j}=$ interaction between maternal and paternal effects.

$R_{k(i j)}=$ effect of $k$ th replicate cross within dam line $\times$ sire line combination.

For $\mathrm{F}_{1}$ productivity, the model equation was:

$Z_{i j k l}=\mu+N_{i}+N_{j}+T_{i j}+M_{j}+P_{i}+K_{i j}+R_{k(i j)}+W_{l(k(i j))}$.

Here, $Z_{\mathrm{ijkl}}$ is the productivity of the l'th female from the $\mathrm{k}^{\prime}$ th replicate cross, and $\mathrm{W}_{\mathrm{l}(\mathrm{k}(\mathrm{ij}))}$ is the residual (within replicate cross) effect of individual $l$.

Estimates of the variances of the terms in equation [1] and [2] ( $\sigma_{n^{\prime}}^{2}$ etc.; see below for interpretations) were obtained by restricted maximum likelihood (REML) using the MIXED procedure in SAS v. 9.1.3. (SAS Institute, 2004), by expressing the covariance between families as linear functions of the variances (see [46], for details). The analysis was performed on a matrix of 342 between-line crosses, excluding all within-line crosses, as required by the bio model $[34,46]$. We tested the one-sided hypotheses that parameter estimates are larger than 0 with likelihood ratio tests, by comparing models where a given

Table I: Observational variance component estimates (SE) and likelihood ratio tests of the null hypothesis $\mathbf{H}_{0}: \sigma^{2}=0$ against $\mathbf{H}_{\mathbf{A}}: \sigma^{2}>$ 0 .

\begin{tabular}{|c|c|c|c|c|c|c|c|c|}
\hline \multirow[b]{2}{*}{ Variance component } & \multicolumn{2}{|c|}{ Lifetime egg production } & \multicolumn{2}{|c|}{ Lifetime offspring production } & \multicolumn{2}{|c|}{ Egg-to-adult survival (arcsine sqrt) } & \multicolumn{2}{|l|}{ FI productivity } \\
\hline & Estimate (SE) & $\mathrm{P}$ & Estimate (SE) & $P$ & Estimate (SE) & $P$ & Estimate (SE) & $P$ \\
\hline$\sigma_{\mathrm{n}}^{2}$ & 0 & - & 0 & - & $0.0012(0.002)$ & 0.12 & $9(3.7)$ & $<0.001$ \\
\hline$\sigma_{t}^{2}$ & $8.5(4.6)$ & 0.015 & $5.1(4.3)$ & 0.055 & $0.0046(0.003)$ & 0.035 & $6.7(3.7)$ & 0.015 \\
\hline$\sigma_{\mathrm{m}}^{2}$ & $12.7(5.3)$ & $<0.001$ & $10.8(4.6)$ & $<0.001$ & $0.003(0.003)$ & 0.035 & $4.3(2.7)$ & 0.004 \\
\hline$\sigma_{\mathrm{p}}^{2}$ & $12.6(5.3)$ & $<0.001$ & $11.5(4.9)$ & $<0.001$ & $0.0043(0.003)$ & 0.01 & 0 & - \\
\hline$\sigma_{k}^{2}$ & $0.2(6.2)$ & 0.5 & $0.5(6)$ & 0.18 & 0 & - & $8.5(4.7)$ & 0.008 \\
\hline$\sigma_{\text {rep }}^{2}$ & $125.7(7.5)$ & $<0.001$ & $121.9(7.2)$ & $<0.001$ & $0.118(0.006)$ & $<0.001$ & $24.1(4.1)$ & $<0.001$ \\
\hline$\sigma_{w}^{2}$ & & & & & & & 116.1 (3.7) & $<0.001$ \\
\hline
\end{tabular}

$\sigma_{\text {rep }}^{2}$ : variance among replicate crosses

$\sigma_{w}^{2}$ : variance among $F_{1}$ females from the same replicate cross 
parameter was set to 0 with a model where all parameters were allowed to assume non-negative values [46]. For the analysis of $F_{1}$ productivity, we included a term to separate the variance among females within replicate crosses from the variance among replicate crosses. This was done by adding the statement "RANDOM REPCROSS (SIRE*DAM);" to the PROC MIXED commands, where "SIRE" and "DAM" refer to the line of the sire and dam, and "REPCROSS" is a class variable. For the other traits there was only one measurement per replicate cross, hence the replicate cross variation is not distinguishable from the residual variation.

The "observational" variance components (denoted by sigma's) have the following interpretations in terms of "causal" components (denoted by $V^{\prime}$ s):

1) $\sigma_{n}^{2}$ : nuclear additive variance. Assuming that our lines represent a random sample from the base population, and that we have estimated $F$ correctly ( $F$ from pedigrees does not always reflect the actual $F$, due to selection for heterozygosity that can occur during the inbreeding process), $\sigma_{n}^{2}$ $=1 / 2 F V_{A}+1 / 4 F^{2} V_{A A}$, where $V_{A}$ and $V_{A A}$ are the additive genetic and additive by additive epistatic variances, respectively, in the base population, and higher order epistasis is ignored for simplicity ([16], equation 15.8). If $V_{A A}$ is assumed to be small, then $V_{A}$ can be estimated as $2 \sigma_{n}^{2} / F$.

2) $\sigma_{t}^{2}$ : nuclear interaction variance. Under the same assumptions as above, $\sigma_{t}^{2}=1 / 2 F^{2} V_{A A}+F^{2} V_{D}+F^{3} V_{A D}+$ $F^{4} V_{D D^{\prime}}$, where $V_{A D}$ and $V_{D D}$ are the additive by dominance and dominance by dominance epistatic variances, respectively. Assuming the epistatic terms are small, the dominance variance $V_{D}$ can be estimated as $\sigma_{t}^{2} / F^{2}$.

3) $\sigma_{m}^{2}$ : maternal effect variance $V_{m}$ including both maternal genotype and common-environment effects, as well as possible interactions or co-variances between maternal nuclear and maternal extra-nuclear effects.

4) $\sigma_{p}^{2}$ : paternal effect variance $V_{p}$, including both paternal genotype and common-environment effects, as well as possible interactions or co-variances between paternal nuclear and paternal extra-nuclear effects.

5) $\sigma_{k}^{2}$ : interaction variance of paternal and maternal effects, and of nuclear and extra-nuclear effects (e.g. cytonuclear), $V_{k}$.

6) $\sigma_{r e p}^{2}$ : variance among replicate crosses within line combinations.

7) $\sigma_{w}^{2}$ : (for $\mathrm{F}_{1}$ productivity only) variance among females within replicate crosses.
Note that neither $\sigma_{r e p}^{2}$ or $\sigma_{w}^{2}$ (or their sum) can be equated with the environmental variance $V_{E^{\prime}}$ because unless the parental lines are fully inbred $(F=1)$, both $\sigma_{\text {rep }}^{2}$ and $\sigma^{2}{ }_{w}$ will have genetic as well as environmental contributions. To estimate $V_{E^{\prime}}$ we subtracted the other causal components of variance from the total phenotypic variance $\left(V_{\text {tot }}\right): V_{E}=V_{\text {tot }}-V_{A}-V_{D}-V_{M}-V_{P}-V_{K} \cdot V_{\text {tot }}$ was estimated as the sum of all the observational components of variance, taking $\sigma_{n}^{2}$ twice (because $N$ appears twice in the model equation).

Coefficients of genetic variation $(\mathrm{CV})$ for each parameter estimate were calculated using the additive genetic coefficient of variation, $\mathrm{CV}_{\mathrm{A}}=100 \sqrt{\mathrm{V}_{\mathrm{A}}} / \bar{X}[21]$ where $\bar{X}$ is the phenotypic trait mean. Coefficients of genetic variation allow comparisons of the sources of phenotypic variation across different traits.

\section{Results}

The partitioning of phenotypic variance into observational genetic variance estimates is given in Table 1 . In the initial diallel cross, variance in lifetime egg production came from highly significant contributions of $\sigma_{m}^{2}$ and $\sigma_{p^{\prime}}^{2}$ representing line-specific maternal and paternal effects, respectively. The strong maternal effect is not surprising, because this term includes the effect of the female's own (inbred) genotype on her egg production. The maternal effect term could also include a contribution of common environment effects shared by different females from the same line. The highly significant paternal effect on egg production, which is of the same magnitude as the maternal effect, is more surprising. This indicates that the genotype of the inbred line males, or the environment shared by different males from the same line, strongly affected egg production by their mates. In contrast, there was no evidence for nuclear additive effects $\left(\sigma_{n}^{2}\right)$ on egg production, and nuclear non-additive effects $\left(\sigma^{2}\right)$, while significant, were smaller than the paternal and maternal effects. These results are not surprising, because we would not expect that the genotype of a female's eggs would have much if any direct influence on the number of eggs she produces. Finally, there was no evidence for non-reciprocal interaction effects $\left(\sigma_{k}^{2}\right)$ on egg production. Variance components and significance levels for lifetime offspring production were similar to those for lifetime egg production (Table 1), reflecting the overall high survival in these crosses (about 80\%).

The proportion surviving in the initial crosses showed no evidence for nuclear additive or non-reciprocal interaction effects, marginally significant nuclear dominance and maternal effects, and a significant paternal effect (Table 1 ). The latter result indicates that some property of male line influenced egg survival, independently of the males' 
genetic contribution to the eggs (the latter would be reflected in $\sigma_{n}^{2}$ and/or $\sigma_{t}^{2}$ ).

Up to six $F_{1}$ females from each initial cross were mated to randomly selected outbred males, and their productivity measured. We treat productivity of these females as a trait of the females themselves, rather than of the cross, as this allows us to investigate the co-expression of female dam and sire genotypes. This approach is justified by the fact that male genotype explained only a small fraction of the productivity variation (see Methods). We found highly significant nuclear additive variance $\left(\sigma_{n}^{2}\right)$ for $\mathrm{F}_{1}$ productivity, and marginally significant nuclear non-additive variance $\left(\sigma_{t}^{2}\right)$. While $\sigma_{m}^{2}$ also contributed significantly to $\mathrm{F}_{1}$ productivity (possibly reflecting cytoplasmic effects), $\sigma_{p}^{2}$ did not. In contrast to the results from the first cross, the non-reciprocal compatibility variance $\sigma^{2}{ }_{k}$ also contributed significantly to the variance in $\mathrm{F}_{1}$ productivity. Finally, there was a highly significant effect of initial replicate cross $\left(\sigma_{r e p}^{2}\right)$, meaning that full-sibs resembled each other in productivity more than non-full sibs from the same cross combination (line $i$ female $\times$ line $j$ male). This likely largely reflects common environment effects, although because the lines were not completely inbred, some genetic divergence between replicate full-sibships may have contributed as well.

Estimates for causal variance components and coefficients of genetic variation are listed in Table 2. The estimates are given mainly for heuristic purposes. They assume that epistatic variance is absent; if present, epistasis would contribute mostly to the estimate for dominance variance, and slightly to the additive variance. The sources of genetic variance in fitness were strikingly different when estimated in different life-history stages. Overall, variance in parental productivity and egg-to-adult survival was dominated by maternal and paternal effects. In contrast, variance in $\mathrm{F}_{1}$ productivity was affected by sizeable components of both additive and non-additive genetic variation as well as by sex-specific interactions between the maternal and paternal genomes. The generally strong interaction effects are illustrated in Figure 1.
As expected, we did not detect any differences in lifetime egg production when comparing within- and betweenline crosses. In contrast, we found strong evidence for heterosis (Table 3) for variables where the maternal and paternal genomes were co-expressed. Heterosis clearly affected juvenile performance, as reflected in both egg-toadult survival as well as $\mathrm{F}_{1}$ productivity. We note that the latter effect, where full co-expression is expected, was stronger than the former (Table 3).

\section{Discussion}

Our analyses revealed sizeable additive and non-additive components of genetic variation for our most comprehensive fitness variable ( $\mathrm{F}_{1}$ productivity) in C. maculatus, illustrating that the diallel cross offers a useful empirical route to provide insights into rather complex aspects of the genetic architecture of fitness. In addition, we found large paternal and maternal effects indicative of direct effects on egg production. We also document striking differences in the genetic architecture of fitness traits measured in different life history stages: while egg-to adult survival was significantly affected by paternal effects, we found considerable additive and non-additive genetic variation for $F_{1}$ productivity. This stresses the importance of obtaining comprehensive measures of fitness when assessing the potential for indirect genetic benefits. Below we discuss these findings in more detail, and use our results to discuss potential processes that may contribute to genetic variance in fitness. Finally, we ask what the implications are of our results in terms of the evolution of female choice by indirect selection.

In general, we found fairly high levels of both additive and non-additive genetic variance in $\mathrm{F}_{1}$ productivity. Our estimate of additive genetic variance for $\mathrm{F}_{1}$ productivity was $14 \%$ expressed as the coefficient of additive genetic variation $C V_{A^{\prime}}$ which lies within the range of $C V_{A}$ of lifetime fitness reported from natural populations of other species (6-44\% for females) $[40,60,61]$. This amount of genetic variation should in theory be sufficient to derive additive genetic benefits of mate choice [62] (see below). One potential concern is that our estimates may be affected by selection when creating distinct genotypes through

Table 2: Estimates of raw and scaled variances and coefficients of genetic variation (CV \%; see Houle 1992).

\begin{tabular}{|c|c|c|c|c|c|c|c|c|c|c|c|c|}
\hline \multirow[b]{2}{*}{ Variance } & \multicolumn{3}{|c|}{ Lifetime egg production } & \multicolumn{3}{|c|}{ Lifetime offspring production } & \multicolumn{3}{|c|}{ Egg-to-adult survival (arcsine sqrt) } & \multicolumn{3}{|c|}{ FI productivity } \\
\hline & Esti-mate & $\%$ & $\mathrm{CV}$ & Esti-mate & $\%$ & $\mathrm{CV}$ & Esti-mate & $\%$ & $\mathrm{CV}$ & Esti-mate & $\%$ & $\mathrm{CV}$ \\
\hline $\mathrm{V}_{\mathrm{A}}$ & 0 & 0 & 0 & 0 & 0 & 0 & 0.0028 & 2.12 & 4.60 & 20.32 & 11.44 & 14.16 \\
\hline$V_{D}^{A}$ & 10.67 & 6.69 & 14.17 & 6.50 & 4.33 & 12.81 & 0.0058 & 4.37 & 6.60 & 8.45 & 4.75 & 9.13 \\
\hline$V_{M}$ & 12.73 & 7.98 & 15.48 & 10.80 & 7.20 & 16.51 & 0.0030 & 2.24 & 4.72 & 4.26 & 2.40 & 6.48 \\
\hline$V_{p}$ & 12.59 & 7.89 & 15.39 & 11.54 & 7.70 & 17.07 & 0.0043 & 3.26 & 5.70 & 0 & 0 & 0 \\
\hline$v_{k}$ & 0.17 & 0.10 & 1.77 & 0.51 & 0.34 & 3.58 & 0 & 0 & 0 & 8.48 & 4.77 & 9.15 \\
\hline$v_{E}$ & 123.45 & 77.35 & 48.20 & 120.58 & 80.42 & 55.17 & 0.1167 & 88.01 & 29.62 & 136.16 & 76.64 & 36.66 \\
\hline
\end{tabular}



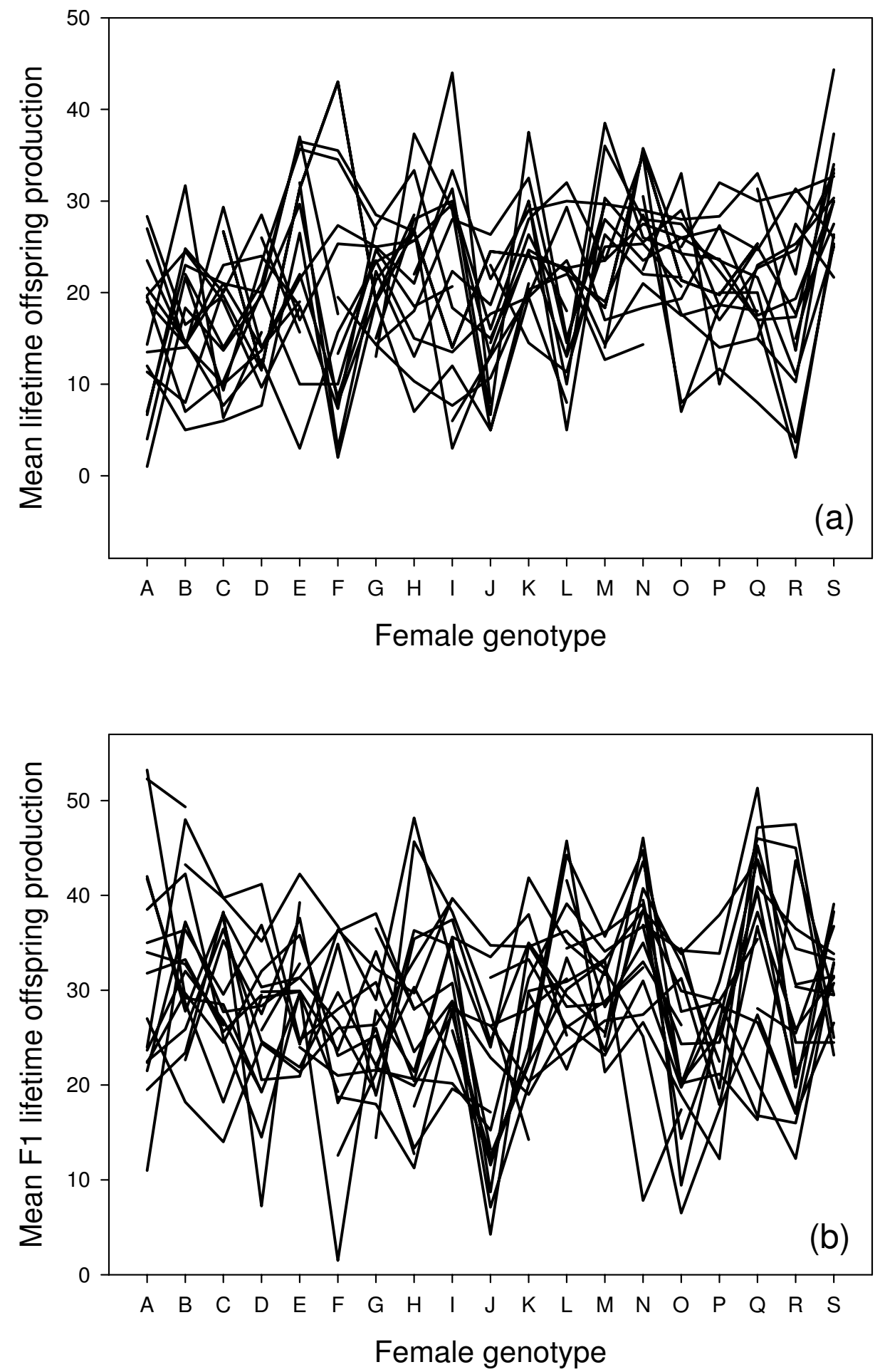

Figure I

Mean (a) lifetime offspring production and (b) mean $F_{1}$ daughter lifetime offspring production for all combinations of female (abscissa) and male (solid lines) genotypes (within-line crosses excluded). Note the extensive amount of interactions between genotypes. 
Table 3: Comparisons of fitness components in within- and between-line crosses (excluding crosses where no eggs where produced).

\begin{tabular}{llll}
\hline Trait & Within-line & Between-line & $F$ - tests of equality of means \\
\hline Lifetime egg production & $23.73(1.53)$ & $23.05(0.4)$ & $F_{1,946}=0.17, P=0.67$ \\
Lifetime offspring production & $16.07(1.48)$ & $19.9(0.4)$ & $\begin{array}{l}F_{1,950}=5.93, P=0.015 \\
F_{1,946}=30.97, P<0.001\end{array}$ \\
Egg-to-adult survival (\%) & $62.01(0.03)$ & $79.75(0.01)$ & $F_{1,2903}=209, P<0.001$ \\
\hline FI productivity & $16.97(0.82)$ & $31.82(0.25)$ & \\
\hline
\end{tabular}

Given are mean (SE) and $F$ - tests of equality of means.

inbreeding, possibly due to the purging of genes with non-additive effects (e.g., recessive lethals). Unless the removal of alleles with non-additive effects caused selective extinction of genotypes with intermediate breeding values, which seems unlikely, this process would tend to decrease estimates of additive genetic variation [63]. Thus, selection during the inbreeding process is unlikely to alone explain the sizeable amount of additive genetic variation for fitness found here (see Methods). Because the continual input from mutations seems unlikely to account for the presence of levels of additive genetic variation for fitness as high as those documented here [31], our study suggests that sources other than mutation contribute to additive genetic variation.

Previous studies employing reciprocal crosses/the bio model have dealt with life history traits of the parental lines (e.g. fecundity) or of the offspring, typically in the juvenile stage $[41-44,64,65]$. Most of these studies report low or moderate levels of additive genetic variation for traits related to parental fitness, but these results are not directly comparable to those reported here. This is because, as shown here, the genetic architecture of fitness may vary across life history traits, and it is imperative to include offspring reproductive success in any inclusive measure of fitness [66-68].

We found significant non-additive variance, $\sigma_{t}^{2}$ suggesting that reciprocal epistatic interactions between nuclear genes and/or dominance significantly affect fitness in our population. There was also a sizeable effect of more complex and sexually non-reciprocal genetic interactions $\left(\sigma_{k}^{2}\right)$ in $\mathrm{F}_{1}$ productivity. Sexually non-reciprocal epistasis may reflect cyto-nuclear genetic interactions, which has been shown to affect fitness related traits in both C. maculatus $[69,70]$ and other insects $[71,72]$. Sexually non-reciprocal interaction could also arise as a result of Wolbachia infections $[73,74]$, but we note that Wolbachia infections has been sought for but never found in C. maculatus [75].

\section{Parental effects}

Both variance in parental environmental effects and nuclear genetic variation among parents will contribute to parental effects. In our case, however, we suggest that parental effects are largely due to genetic variation, because rearing conditions were highly standardized and thus uniform across lines. Parental effects were large for lifetime egg and offspring production but lower for eggto-adult survival and $\mathrm{F} 1$ productivity (Table 1 ). The former measure primarily reflects variance in fertility across lines, while the latter measure will be affected by the co-expression of the two parental haploid genomes. We note here that sire families that gave rise to high egg production by their mates also gave rise to higher survival of the offspring (unpublished data), suggesting that some property of the male ejaculate (see below) influenced both egg production and survival of the eggs. These facts underscore the point that studies of the genetic architecture of fitness should focus on comprehensive measures of offspring adult performance.

Sizeable maternal effects, both genetic and environmental, are well documented in C. maculatus [76-78]. Furthermore, paternal effects are also pronounced in this taxa, mediated by seminal fluids in the large ejaculate (approximately $8 \%$ of male body weight) [79], and our results reflect this fact. Nutrients in the male ejaculate have been shown to be incorporated into reproductive and somatic tissue in female Bruchid beetles [80] and females that receive larger ejaculates show elevated egg production $[79,81,82]$. There is growing evidence that ejaculate volume shows additive genetic variation and it is often positively correlated with body size or male condition in insects $[51,79,83,84]$. For example, nutrient investment and spermatophore size in the butterfly Pieris napi is heritable, and male genotype influence female fecundity and longevity [85]. This suggests that females, by mating with males with large ejaculates, will derive direct fecundity and survival benefits as well as indirect genetic benefits. Such indirect benefits may, however, be negated by sexually antagonistic genes (discussed below).

\section{Implications for indirect sexual selection}

The presence of additive genetic variance provides the raw material for indirect selection by the good genes process $[2,3,14,17]$. The amount of standing additive genetic variation for fitness present in our population should in principle be sufficient to generate indirect selection on female 
choice [12]. Further, genetic variation for fitness in our population was also influenced by genes with non-additive effects, and the raw material for females deriving indirect benefits by mate compatibility is thus also present. However, while non-additive genetic effects may be ubiquitous $[28,86]$, the role of these effects in driving the evolution of mating biases is both debated $[5,15,41,87]$ and somewhat misconstrued. Models of the evolution of female choice have shown that genes with non-additive effects do not contribute to indirect selection on female choice (see Introduction), so any selection on female choice for genetic compatibility benefits must be direct. It is also worth noting that in order for females to gain indirect benefits by choosing compatible mates, females need to assess their own genome, assess the male genome, and predict the genetic compatibility effects on offspring fitness. The latter also requires taking into account recombination, asymmetric inheritance patterns and sex-specific effects [88]. Although this may seem highly unlikely, female choice for compatible males may nevertheless occur for specific loci or more restricted regions of the genome, such as the major histocompatibility complex (MHC) in vertebrates [89].

In general, the presence of genetic variation for fitness has been interpreted as supporting a role for indirect genetic benefits in the evolution of female choice [62]. However, three points call this interpretation into question. First, when indirect selection does occur, the efficacy of indirect selection may nevertheless be low in the face of direct selection $[12,14]$. Direct selection on female preference is probably generally underestimated in empirical research $[90,91]$. In our model system, direct selection on female choice is likely to be strong $[92,93]$ and thus may override indirect selection. However, Fricke and Arnqvist (2007) showed that sexual selection accelerated adaptation to a novel environment in C. maculatus, suggesting a role for indirect selection under at least some conditions. Second, indirect selection on female choice arises as a result of linkage disequilibrium between female choice/preference genes and genes with additive effects that confer high fitness in offspring [12]. In order for such linkage disequilibrium to build up, even in the presence of additive genetic variation for fitness, females with high level of preference must consistently choose males with a high breeding value for fitness. Very few empirical studies have looked for the consistency of such associations [67]. This problem is aggravated when considering direct selection for indirect benefits in the form of mate compatibility. Here, demonstrations of epistatic fitness variation in conjunction with male $\times$ female interactions in female choice are insufficient: females must also be able to consistently bias fertilization success among males towards males that carry genes that are compatible with her own genes. Empirical evidence for such associations are restricted to the study of MHC in vertebrates [89] and some studies of the deleterious effects of inbreeding [94,95].

Third, perhaps the most fundamental problem in assessing the potential for indirect genetic benefits in sexual selection lies in estimating net selection on female choice [90]. In order to measure paternal genetic contribution to fitness it is vital to obtain proper estimates of offspring genetic quality, preferably the total number of grandchildren produced $[7,60,96]$. Fitness correlates such as fecundity and lifetime offspring production are often reported and assumed to correlate positively with net fitness, but life-history theory and empirical evidence provide little basis for expecting strong positive correlations between individual fitness components and overall genetic quality (i.e., breeding value for fitness) [66-68]. Further, recent empirical research has revealed a negative, or a non-existing, genetic correlation between male and female fitness, suggesting that potential indirect benefits might be nullified through sexually antagonistic genes $[60,61,66,97,98]$. This points to the necessity of measuring the breeding value for fitness in offspring of both sexes, when assessing the potential role of indirect benefits for the evolution of mating biases. Such investigations are currently in progress in C. maculatus.

\section{Conclusion}

In conclusion, we found significant standing genetic variance for fitness related traits in our study population of $C$. maculatus, in sufficient amounts for females to potentially derive indirect genetic benefits of mate choice. The genetic architecture of fitness traits varied dramatically with the life history stage in which fitness traits were measured. Parental effects, in particular paternal effects, were significant in the juvenile stage, while sizeable additive and nonadditive effects were seen in the lifetime reproductive success of $F_{1}$ daughters. Our results support the notion that comprehensive fitness measures should include offspring breeding value. Although our results show that there is a potential for females to derive indirect genetic benefits from mate choice, we note that several facts may nevertheless make selection for indirect genetic benefits ineffectual. These include direct selection, lack of accuracy in female choice and sexually antagonistic genetic variation.

\section{Authors' contributions}

TB participated in the design of the study, collected data, performed statistical analyses and wrote the paper. UF and AM participated in the design of the study and collected data. JF participated in the statistical analyses and writing of the paper. GA participated in the design of the study and the writing of the paper. All authors read and approved the final manuscript. 


\section{Acknowledgements}

N. Schilling, E. Bomholt and C. Tuni provided invaluable help in data collection. T. Ivy generously provided the SAS code and advice on the initial statistical analysis. A.C. Sørensen provided helpful comments on the manuscript. This work was supported by a Marie Curie Fellowship (MC MEIF-CT-2006-023645) and the Carlsberg Foundation (TB), Wenner-Gren Foundations Fellowship and a stipend from Lennander's Foundation (UF), Marie Curie Fellowship (MC MEIF-CT-2003-50589I) and ARC DP0774587 (AAM) and the Swedish Research Council (to GA).

\section{References}

I. Maynard Smith J: Sexual Selection - A Classification of Models. In Sexual Selection: Testing the Alternatives Edited by: Bradbury JW, Andersson M. New York: John Wiley \& Sons; 1987.

2. Andersson M: Sexual Selection Princeton, New Jersey: Princeton University Press; 1994.

3. Jennions MD, Petrie M: Why do females mate multiply? A review of the genetic benefits. Biological Reviews 2000, 75:21-64.

4. Tregenza T, Wedell N: Genetic compatibility, mate choice and patterns of parentage: Invited review. Molecular Ecology 2000, 9:1013-1027.

5. Zeh JA, Zeh DW: Reproductive mode and the genetic benefits of polyandry. Animal Behaviour 2001, 6 I:105I-1063.

6. Zeh JA, Zeh DW: Toward a new sexual selection paradigm: Polyandry, conflict and incompatibility (Invited article). Ethology 2003, 109:929-950.

7. Hunt J, Bussiere LF, Jennions MD, Brooks R: What is genetic quality? Trends in Ecology \& Evolution 2004, 19:329-333.

8. Eberhard WG: Female control : sexual selection by cryptic female choice Princeton, NJ: Princeton University Press; 1996.

9. Zeh JA, Zeh DW: The evolution of polyandry I: Intragenomic conflict and genetic incompatibility. Proceedings of the Royal Society of London Series B-Biological Sciences 1996, 263:171 I-I7I7.

10. Zeh JA, Zeh DW: The evolution of polyandry .2. Post-copulatory defences against genetic incompatibility. Proceedings of the Royal Society of London Series B-Biological Sciences 1997, 264:69-75.

II. Bradbury JW, Andersson MB: Sexual selection: testing the alternatives New York: John Wiley \& Sons; 1987.

12. Kirkpatrick $M$, Barton NH: The strength of indirect selection on female mating preferences. Proceedings of the National Academy of Sciences of the United States of America 1997, 94: I 282-1286.

13. Houle D, Kondrashov AS: Coevolution of costly mate choice and condition-dependent display of good genes. Proceedings of the Royal Society of London Series B-Biological Sciences 2002, 269:97-I 04.

14. Kirkpatrick M: Good genes and direct selection in evolution of mating preferences. Evolution I996, 50:2125-2140.

I5. Puurtinen M, Ketola T, Kotiaho JS: Genetic compatibility and sexual selection. Trends in Ecology \& Evolution 2005, 20: I57-1 58

16. Falconer DS, Mackay TFC: Introduction to Quantitative Genetics London: Longman; 1996.

17. Neff BD, Pitcher TE: Genetic quality and sexual selection: an integrated framework for good genes and compatible genes. Molecular Ecology 2005, I 4:19-38.

18. Fisher RA: The Genetical Theory of Natural Selection Oxford: Oxford University Press; 1930.

19. Jones JS: The Heritability of Fitness - Bad-News for Good Genes. Trends in Ecology \& Evolution 1987, 2:35-38.

20. Charlesworth B: The heritability of fitness. In Sexual selection: testing the alternatives Edited by: Bradbury JWaA MB. New York: Wiley; 1987.

21. Houle D: Comparing Evolvability and Variability of Quantitative Traits. Genetics 1992, 130:195-204.

22. Gustafsson L: Lifetime Reproductive Success and Heritability - Empirical Support for Fisher Fundamental Theorem. American Naturalist I 986, I 28:76 |-764.

23. Mousseau TA, Roff DA: Natural-Selection and the Heritability of Fitness Components. Heredity 1987, 59: I8I-I97.

24. Roff DA, Mousseau TA: Quantitative Genetics and Fitness Lessons from Drosophila. Heredity 1987, 58: I03-I I8.

25. Barton NH, Turelli M: Evolutionary Quantitative Genetics How Little Do We Know. Annual Review of Genetics 1989, 23:337-370.

26. Merila J, Sheldon BC: Lifetime reproductive success and heritability in nature. American Naturalist 2000, I 55:301-310.
27. Price T, Schluter D: On the Low Heritability of Life-History Traits. Evolution 1991, 45:853-86I

28. Roff DA, Emerson K: Epistasis and dominance: Evidence for differential effects in life-history versus morphological traits. Evolution 2006, 60:1981-1990.

29. Messina FJ: Heritability and Evolvability of Fitness Components in Callosobruchus-Maculatus. Heredity 1993, 71:623-629.

30. Rowe L, Houle D: The lek paradox and the capture of genetic variance by condition dependent traits. Proceedings of the Royal Society of London Series B-Biological Sciences 1996, 263:I4I5-I42I.

31. Houle D, Morikawa B, Lynch M: Comparing mutational variabilities. Genetics 1996, I43:|467-|483.

32. Rice WR: Heritable Variation in Fitness as a Prerequisite for Adaptive Female Choice - the Effect of Mutation-Selection Balance. Evolution 1988, 42:817-820.

33. Armbruster P, Bradshaw WE, Holzapfel CM: Evolution of the genetic architecture underlying fitness in the pitcher-plant mosquito, Wyeomyia smithii. Evolution 1997, 5 I :45 I-458.

34. Lynch M, Walsh B: Genetics and Analysis of Quantitative Traits Sunderland Massachusetts: Sinauer Associates Inc; 1998.

35. Wolf JB, Brodie ED III, Wade MJ, (Eds): Epistasis and the evolutionary process. Oxford, UK: Oxford University Press; $200 \mathrm{I}$.

36. Burt A: Perspective - the Evolution of Fitness. Evolution 1995, 49: $1-8$.

37. Fowler K, Semple C, Barton NH, Partridge L: Genetic variation for total fitness in Drosophila melanogaster. Proceedings of the Royal Society of London Series B-Biological Sciences 1997, 264:191-199.

38. Arnqvist G, Rowe L: Sexual Conflict Princeton, New Jersey: Princeton University Press; 2005.

39. Kruuk LEB, Clutton-Brock TH, Slate J, Pemberton JM, Brotherstone $S$, Guinness FE: Heritability of fitness in a wild mammal population. Proceedings of the National Academy of Sciences of the United States of America 2000, 97:698-703.

40. McCleery RH, Pettifor RA, Armbruster P, Meyer K, Sheldon BC, Perrins $C M$ : Components of variance underlying fitness in a natural population of the great tit Parus major. American Naturalist 2004, I 64:E62-E72.

4I. Ivy TM: Good genes, genetic compatibility and the evolution of polyandry: use of the diallel cross to address competing hypotheses. Journal of Evolutionary Biology 2007, 20:479-487.

42. Gebhardt MD, Stearns SC: Phenotypic Plasticity for Life-History Traits in Drosophila-Melanogaster .3. Effect of the Environment on Genetic-Parameters. Genetical Research 1992 , 60:87-101.

43. Tatar M, Promislow DEI, Khazaeli AA, Curtsinger JW: Age-specific patterns of genetic variance in Drosophila melanogaster .2. Fecundity and its genetic covariance with age-specific mortality. Genetics 1996, 143:849-858.

44. Telonis-Scott M, Mclntyre LM, Wayne ML: Genetic architecture of two fitness-related traits in Drosophila melanogaster: ovariole number and thorax length. Genetica 2005, I 25:2| |-222.

45. Cockerham CC, Weir BS: Quadratic Analyses of Reciprocal Crosses. Biometrics 1977, 33:187-203.

46. Fry JD: Estimation of genetic variances and covariances by restricted maximum likelihood using PROC MIXED. In Genetic Analysis of Complex Traits using SAS Edited by: Saxton A, Cary NC. SAS Institute Inc; 2004: I I-34.

47. Good-Avila SV, Stephenson AG: Parental effects in a partially self-incompatible herb Campanula rapunculoides L. (Campanulaceae): Influence of variation in the strength of selfincompatibility on seed set and progeny performance. American Naturalist 2003, 161:615-630.

48. Juenger T, Morton TC, Miller RE, Bergelson J: Scarlet gilia resistance to insect herbivory: the effects of early season browsing, plant apparency, and phytochemistry on patterns of seed fly attack. Evolutionary Ecology 2005, I 9:79-101.

49. Byers DL, Platenkamp GAJ, Shaw RG: Variation in seed characters in Nemophila menziesii: Evidence of a genetic basis for maternal effect. Evolution 1997, 5 I: | 445- | 456.

50. Arnqvist $G$, Nilsson $T$, Katvala $M$ : Mating rate and fitness in female bean weevils. Behavioral Ecology 2005, I 6: I23-127.

51. Czesak ME, Fox CW: Genetic variation in male effects on female reproduction and the genetic covariance between the sexes. Evolution 2003, 57:1359-1366. 
52. Fricke C, Arnqvist G: Divergence in replicated phylogenies: the evolution of partial post-mating prezygotic isolation in bean weevils. Journal of Evolutionary Biology 2004, I 7: I 345-1354.

53. Fricke $\mathrm{C}$, Arnqvist $\mathrm{G}$, Amaro N: Female modulation of reproductive rate and its role in postmating prezygotic isolation in Callosobruchus maculatus. Functional Ecology 2006, 20:360-368.

54. Wilson N, Tubman SC, Eady PE, Robertson GW: Female genotype affects male success in sperm competition. Proceedings of the Royal Society of London Series B-Biological Sciences 1997, 264: | 499|-| 495.

55. Kokko H, Jennions MD, Brooks R: Unifying and testing models of sexual selection. Annual Review of Ecology Evolution and Systematics 2006, 37:43-66.

56. Messina FJ, Karren ME: Adaptation to a novel host modifies host discrimination by the seed beetle Callosobruchus maculatus. Animal Behaviour 2003, 65:50I-507.

57. Fox CW, Stillwell RC, Amarillo AR, Czesak ME, Messina FJ: Genetic architecture of population differences in oviposition behaviour of the seed beetle Callosobruchus maculatus. Journal of Evolutionary Biology 2004, I7: I I4I-I I5I.

58. Wade MJ: Genetic Variance for Rate of Population Increase in Natural-Populations of Flour Beetles, Tribolium Spp. Evolution 199I, 45: I574-I584.

59. Mitchell R: Evolution of Oviposition Tactics in Bean Weevil, Callosobruchus-Maculatus (F). Ecology 1975, 56:696-702.

60. Brommer JE: The Intersexual Genetic Correlation for Lifetime Fitness in the Wild and Its Implications for Sexual Selection. PLoS ONE 2007, 2:e744.

6I. Foerster K, Coulson T, Sheldon BC, Pemberton JM, Clutton-Brock $\mathrm{TH}$, Kruuk LEB: Sexually antagonistic genetic variation for fitness in red deer. Nature 2007, 447: I 107-1110.

62. Moller AP, Alatalo RV: Good-genes effects in sexual selection. Proceedings of the Royal Society of London Series B-Biological Sciences 1999, 266:85-91.

63. Wade MJ, Shuster SM, Stevens L: Inbreeding: Its effect on response to selection for pupal weight and the heritable variance in fitness in the flour beetle, Tribolium castaneum. Evolution 1996, 50:723-733.

64. Marshall DJ, Evans JP: The benefits of polyandry in the freespawning polychaete Galeolaria caespitosa. Journal of Evolutionary Biology 2005, 18:735-74I.

65. Evans JP, Garcia-Gonzalez F, Marshall DJ: Sources of genetic and phenotypic variance in fertilization rates and larval traits in a sea urchin. Evolution 2007, 61:2832-2838.

66. Chippindale AK, Gibson JR, Rice WR: Negative genetic correlation for adult fitness between sexes reveals ontogenetic conflict in Drosophila. Proceedings of the National Academy of Sciences of the United States of America 200I, 98: 167I-1675.

67. Head ML, Hunt J, Jennions MD, Brooks R: The indirect benefits of mating with attractive males outweigh the direct costs. Plos Biology 2005, 3:289-294.

68. Roff DA: Life History Evolution Sunderland, MA: Sinauer Associates; 2002.

69. Dowling DK, Abiega KC, Arnqvist G: Temperature-specific outcomes of cytoplasmic-nuclear interactions on egg-to-adult development time in seed beetles. Evolution 2007, 61:194-201.

70. Dowling DK, Nowostawski AL, Arnqvist G: Effects of cytoplasmic genes on sperm viability and sperm morphology in a seed beetle: implications for sperm competition theory? Journal of Evolutionary Biology 2007, 20:358-368.

71. Dowling DK, Friberg U, Hailer F, Arnqvist G: Intergenomic epistasis for fitness: Within-population interactions between cytoplasmic and nuclear genes in Drosophila melanogaster. Genetics 2007, I75:235-244.

72. Rand DM, Clark AG, Kann LM: Sexually antagonistic cytonuclear fitness interactions in Drosophila melanogaster. Genetics 200I, 159:173-187.

73. Rousset F, Bouchon D, Pintureau B, Juchault P, Solignac M: Wolbachia Endosymbionts Responsible for Various Alterations of Sexuality in Arthropods. Proceedings of the Royal Society of London Series B-Biological Sciences 1992, 250:91-98.

74. Charlat $\mathrm{S}$, Hurst GDD, Mercot $\mathrm{H}$ : Evolutionary consequences of Wolbachia infections. Trends in Genetics 2003, 19:217-223.

75. Tuda M, Ronn J, Buranapanichpan S, Wasano N, Arnqvist G: Evolutionary diversification of the bean beetle genus Callosobruchus (Coleoptera : Bruchidae): traits associated with storedproduct pest status. Molecular Ecology 2006, I5:354I-355 I.
76. Fox CW: Maternal and Genetic Influences on Egg Size and Larval Performance in a Seed Beetle (Callosobruchus-Maculatus) - Multigenerational Transmission of a Maternal Effect. Heredity 1994, 73:509-5I7.

77. Fox CW, Czesak ME, Wallin WG: Complex genetic architecture of population differences in adult lifespan of a beetle: nonadditive inheritance, gender differences, body size and a large maternal effect. Journal of Evolutionary Biology 2004, 17:1007-1017.

78. Fox CW, Savalli UM: Inheritance of environmental variation in body size: Superparasitism of seeds affects progeny and grandprogeny body size via a nongenetic maternal effect. Evolution 1998, 52:172-182.

79. Savalli UM, Fox CW: Genetic variation in paternal investment in a seed beetle. Animal Behaviour 1998, 56:953-96I.

80. Boucher L, Huignard J: Transfer of Male Secretions from the Spermatophore to the Female Insect in Caryedon-Serratus (OI) - Analysis of the Possible Trophic Role of These Secretions. Journal of Insect Physiology 1987, 33:949.

81. Fox CW, Stillwell RC, Wallin WG, Hitchcock LJ: Temperature and host species affect nuptial gift size in a seed-feeding beetle. Functional Ecology 2006, 20: 1003-10II

82. Rönn J, Katvala M, Arnqvist G: Interspecific variation in ejaculate allocation and associated effects on female fitness in seed beetles. J Evol Biol 2008, 2I(2):46I-470.

83. Simmons LW, Kotiaho JS: Evolution of ejaculates: Patterns of phenotypic and genotypic variation and condition dependence in sperm competition traits. Evolution 2002, 56:1622-1631.

84. Garcia-Gonzalez F, Simmons LW: Paternal indirect genetic effects on offspring viability and the benefits of polyandry. Current Biology 2007, 17:32-36.

85. Wedell N: Male genotype affects female fitness in a paternally investing species. Evolution 2006, 60:1638-1645

86. Crnokrak P, Roff DA: Dominance Variance - Associations with Selection and Fitness. Heredity 1995, 75:530-540.

87. Kotiaho JS, Lebas NR, Puurtinen M, Tomkins JL: On the resolution of the lek paradox. Trends in Ecology \& Evolution 2008, 23:I-3.

88. Charlesworth B, Coyne JA, Barton NH: The Relative Rates of Evolution of Sex-Chromosomes and Autosomes. American Naturalist 1987, 130: I I3-|46.

89. Penn DJ: The scent of genetic compatibility: Sexual selection and the major histocompatibility complex. Ethology 2002, I08: |-2|.

90. Arnqvist $G$, Kirkpatrick $M$ : The evolution of infidelity in socially monogamous passerines: The strength of direct and indirect selection on extrapair copulation behavior in females. American Naturalist 2005, 165:S26-S37.

91. Kotiaho JS, Puurtinen M: Mate choice for indirect genetic benefits: scrutiny of the current paradigm. Functional Ecology 2007, $21: 638-644$

92. Ronn J, Katvala M, Arnqvist G: Coevolution between harmful male genitalia and female resistance in seed beetles. Proceedings of the National Academy of Sciences of the United States of America 2007, 104:10921-10925.

93. Ronn J, Katvala M, Arnqvist G: The costs of mating and egg production in Callosobruchus seed beetles. Animal Behaviour 2006 72:335-342.

94. Tregenza T, Wedell N: Polyandrous females avoid costs of inbreeding. Nature 2002, 4I5:7|-73.

95. Zeh JA, Zeh DW: Outbred embryos rescue inbred half-siblings in mixed-paternity broods of live-bearing females. Nature 2006, 439:20I-203

96. Kokko H, Brooks R, Jennions MD, Morley J: The evolution of mate choice and mating biases. Proceedings of the Royal Society of London Series B-Biological Sciences 2003, 270:653-664.

97. Gibson JR, Chippindale AK, Rice WR: The $\mathbf{X}$ chromosome is a hot spot for sexually antagonistic fitness variation. Proceedings of the Royal Society of London Series B-Biological Sciences 2002, 269:499-505

98. Pischedda $A$, Chippindale AK: Intralocus sexual conflict diminishes the benefits of sexual selection. Plos Biology 2006, 4:2099-2103. 